\title{
Preferências de pessoas idosas pela informação prognóstica numa situação de doença grave, com menos de um ano de vida
}

\author{
Older people's preferences for prognostic information in a situation \\ of serious illness with less than a year to live
}

Rafaela Jorge (https://orcid.org/0000-0001-7726-5523) ${ }^{1}$

Andreia Teixeira (http://orcid.org/0000-0003-1199-2220) ${ }^{2}$

Natália Calanzani (https://orcid.org/0000-0002-5068-2543) ${ }^{3}$

Rui Nunes (https://orcid.org/0000-0002-1377-9899) ${ }^{4}$

Liliana Sousa (https://orcid.org/0000-0003-3491-7119) ${ }^{5}$

${ }^{1}$ Programa Doutoral em Geriatria e Gerontologia, Departamento de Educação e Psicologia, Universidade de Aveiro. Campus

Universitário de Santiago. 3810-193 Aveiro Portugal. rafaelajorge@outlook.com

${ }^{2}$ Centro de Investigação em Tecnologias e Serviços de Saúde (CINTESIS). Porto Portugal.

${ }^{3}$ Instituto Usher de Ciências da Saúde da População e Informática, Centro de Ciências da Saúde da População, Universidade de Edimburgo. Edimburgo Reino Unido.

${ }^{4}$ Faculdade de Medicina da Universidade do Porto. Porto Portugal.

${ }^{5}$ Departamento de Educação e Psicologia, Centro de Investigação em Tecnologias e Serviços de Saúde (Cintesis.ua), Universidade de Aveiro. Aveiro Portugal.

\begin{abstract}
This study aimed to determine the preferences of community-dwelling older people about information disclosure regarding poor prognosis, the likely symptoms and problems, and the care options available in a situation of serious illness with less than a year to live; and to identify factors associated with a preference for information disclosure regarding poor prognosis. The Brazilian version of the Preferences and Priorities for End of Life Care (PRISMA) questionnaire was administered face-to-face to 400 older people, living in the city of Belo Horizonte, Minas Gerais, Brazil. The main results indicated that $74.0 \%$ preferred to be informed that they had limited time left, $89.3 \%$ wished to be informed about symptoms and problems, and 96.3\% about available care options. The factors associated with preferences for information about poor prognosis were: gender (women: $O R=0.446,95 \%$ CI: 0.269-0.738) and choosing the least preferred place to die (home of a relative or friend: $O R=$ 2.423, 95\% CI: 1.130-5.198. These results show that most older people want to be informed in an advanced illness situation with less than a year to live. Health care professionals need to be prepared to anticipate news about poor prognosis and the disease.
\end{abstract}

Key words Aged, Palliative care, Information, Prognosis, Brazil.
Resumo O objetivo deste estudo foi determinar as preferências de pessoas idosas, residentes na comunidade, pela divulgação de informações relativas ao tempo de vida limitado, sintomas e problemas, e opções disponíveis para cuidados numa situação de doença grave, com menos de um ano de vida; e identificar os fatores associados com a preferência pela informação sobre prognóstico de vida limitado. Foi aplicado a versão brasileira do questionário sobre Preferências e Prioridades para os Cuidados de Fim de Vida (PRISMA), por meio de entrevista face-a-face, a 400 pessoas idosas, residentes na cidade de Belo Horizonte, Minas Gerais, Brasil. Os principais resultados indicam que: $74.0 \%$ preferem ser informados sobre o tempo de vida limitado, $89.3 \%$ sobre os sintomas e problemas e $96.3 \%$ acerca das opções de cuidados. Os fatores associados à preferência pela informação sobre prognóstico de vida limitado foram: gênero (mulheres: OR=0.446, 95\% IC:0.269-0.738) e local menos preferido de morte (casa de um familiar ou amigo: $O R=2.423,95 \%$ IC:1.130-5.198). Esses resultados mostram que a maioria das pessoas idosas deseja ser informada numa situação de doença avançada, com menos de um ano de vida. Os profissionais de saúde precisam estar preparados para antecipar notícias sobre o prognóstico de vida limitado e a doença.

Palavras-chave Idoso, Cuidados paliativos, Informação, Prognóstico, Brasil. 


\section{Introdução}

Com a maioria das pessoas enfrentando um período de doença terminal antes da morte ${ }^{1,2}$, é frequente os profissionais de saúde precisarem comunicar más notícias sobre o diagnóstico e prognóstico aos pacientes ${ }^{3-5}$. Contudo, informar sobre uma situação de terminalidade de vida ainda é uma tarefa difícil para muitos profissionais da saúde ${ }^{3,5,6}$; por isso, é frequente ocorrer a omissão parcial ou total de informações sobre a doença, ou então dar a informação à família e não ao paciente p-9 $^{7-9}$ literatura aponta várias razões para os médicos relutarem em dar informações prognósticas para os pacientes, tais como: falta de formação adequada em comunicação de notícias difíceis, dificuldades de aceitar não ser possível oferecer cura ao paciente, receio das reações dos pacientes e/ou dos familiares e incertezas sobre o prognóstico da doença $a^{3,4,10,11}$. No entanto, a informação é fundamental para aqueles que enfrentam uma doença grave e avançada e a compreensão das preferências do paciente em relação às informações possibilita uma resposta adequada dos profissionais ${ }^{3,10,12}$. A informação, devidamente esclarecida, pode ajudar o paciente e familiares a tomar as decisões sobre os tratamentos, definir metas realistas, ajudar no seu planejamento de vida e aumentar a esperança ao invés de diminuí-la ${ }^{4,13}$. Além disso, quando o paciente recebe menos informações é mais provável que as suas preferências em fim de vida não sejam atendidas $^{14}$, sendo mais propensos a receber cuidados agressivos antes da morte ${ }^{4,15}$.

Além disso, há evidências de que a maioria das pessoas, de diferentes culturas, quando questionada, prefere ser sempre informada sobre o diagnóstico, seja físico ou psiquiátrico; e uma proporção menor, mas substancial, quer saber informações prognósticas detalhadas, embora menos quando esta informação não é favorá$\mathrm{vel}^{3,7,8,12,16-18}$. Um estudo europeu de base populacional mostrou que numa situação hipotética de doença grave, como o câncer, e com menos de um ano de vida, $73.9 \%$ dos participantes preferem ser sempre informados sobre o tempo de vida limitado ${ }^{3}$. Além disso, $81.3 \%$ dos participantes gostariam de ser sempre informados sobre os sintomas e os problemas e $89.5 \%$ preferem ser sempre informados sobre as opções de cuidados (serviços disponíveis, lugares onde poderia receber assistência, tratamentos e medicação) ${ }^{3}$.

Outro estudo com pacientes de dois hospitais de câncer de São Paulo (Brasil) e médicos especialistas em oncologia, que atuam em todo o Brasil, mostrou que $92 \%$ dos pacientes acham que todos os pacientes devem saber sobre o estágio terminal da doença ${ }^{7}$. Esse estudo também demonstra a relutância de alguns médicos e familiares em fornecer informação. Os dados mostram que 18.3\% dos médicos e $20.8 \%$ dos familiares acham que nem todos os pacientes devem saber sobre a fase terminal da doença, em comparação com $8 \%$ dos pacientes ${ }^{7}$. Esses dados revelam que alguns médicos adotam uma relação mais paternalista com os pacientes e alguns familiares podem criar barreiras na transmissão de informações sobre a fase terminal da doença para o paciente ${ }^{7}$.

As preferências das pessoas idosas por informações prognósticas são de particular interesse, pois é o grupo populacional que mais enfrenta doenças graves em estágio avançado e que precisa tomar decisões mais complexas ${ }^{19,20}$. Além disso, a idade avançada de pacientes com câncer é considerada um dos fatores de risco para receber menos informação sobre o prognóstico ${ }^{9,21}$. Estudos anteriores mostram que geralmente os pacientes idosos com câncer ${ }^{21-23}$ querem ser totalmente informados numa situação de doença que ameaça à continuidade da vida. No entanto, também existem estudos que mostram uma maioria de pacientes idosos com câncer com uma preferência por não querer ser informados sobre a expectativa de vida ${ }^{14}$ ou por não querer ser totalmente informados sobre a doença ${ }^{24}$. Além disso, as pessoas idosas preferem receber mais informação sobre o diagnóstico do que o prognóstico ${ }^{14,25,26} \mathrm{e}$ querem saber menos informações do que os mais jovens ${ }^{14,24,27}$. Um estudo europeu de base populacional mostrou que as pessoas com idade $<70$ anos foram mais propensas a querer saber sobre o tempo de vida limitado ${ }^{3}$. Na Bélgica, outro estudo de base populacional, mostrou que os participantes mais jovens (com idade entre 25-34, 3544 e 45-54 anos) foram mais prováveis de querer ser totalmente informados sobre a doença ${ }^{12}$.

A maioria dos estudos sobre as preferências por informações são focados principalmente em pacientes com câncer e na fase final da vida ${ }^{12}$. Alguns países têm realizado estudos de base populacional sobre as preferências em fim de vida, incluindo sobre informações, para tomar decisões de políticas públicas, tais como, o desenvolvimento de programas educacionais para conscientizar a população e profissionais da saúde sobre essas questões ${ }^{12,28}$. No Brasil, apesar do avanço nos últimos anos dos cuidados paliativos, pouco se sabe sobre as preferências em fim de vida da população, incluindo sobre informações prognósticas. Sendo assim, torna-se fundamental 
realizar estudos sobre a opinião das pessoas em relação às suas preferências para conscientizar os profissionais da saúde sobre a importância da inclusão do respeito às vontades dos pacientes nos cuidados em fim de vida.

O objetivo deste estudo é conhecer as preferências de pessoas idosas, residentes na comunidade, por informações sobre o seu tempo de vida limitado, sintomas e problemas e opções disponíveis para cuidados numa situação de doença grave, com menos de um ano de vida; e examinar a influência dos fatores sociodemográficos na preferência por ser sempre informado sobre o tempo de vida limitado.

\section{Métodos}

Trata-se de um estudo transversal, realizado entre fevereiro e julho de 2015 . O universo do estudo é definido pelos indivíduos com idade $\geq 60$ anos, de ambos os sexos, residentes no município de Belo Horizonte, Minas Gerais (MG), Brasil. A primeira etapa para a definição da amostra deste estudo consistiu em consultar no Censo Demográfico de 2010, do Instituto Brasileiro de Geografia Estatística (IBGE), o tamanho da população idosa de Belo Horizonte. Após verificarmos que o total dessa população é de 299.177 pessoas, seguimos para a segunda etapa que consistiu em calcular o tamanho da amostra. Após consultar a tabela Krejcie and Morgan ${ }^{29}$ verificou-se que o tamanho da amostra necessária para ser representativa da população idosa de Belo Horizonte, com um nível de confiança de 95\%, considerando o cenário mais conservador $(p=0.5)$ e uma margem de erro de $5 \%$, é de, aproximadamente, 400 indivíduos. Na terceira etapa considerou-se a distribuição por grupo etário (60-69 anos; 7079 anos; $\geq 80$ anos) e gênero da população idosa da cidade de Belo Horizonte para o cálculo das subamostras $^{30}$. O número de pessoas idosas em cada estrato foi calculado, utilizando o método de amostragem estratificada, de uma forma proporcional, de modo a garantir que a distribuição de participantes amostrados por idade e gênero fosse correspondente à distribuição real da população.

A coleta dos dados decorreu no Centro de Referência da Pessoa Idosa (CRPI), vinculado à Secretaria Municipal Adjunta de Direitos da Cidadania (SMADC) e nos grupos de convivência de idosos do Serviço de Convivência e Fortalecimento de Vínculos do Centro de Referência de Assistência Social (CRAS), gerenciado pela
Secretaria Municipal Adjunta de Assistência Social (SMAAS). Foram selecionados pela SMAAS dez grupos de convivência de idosos do CRAS, de forma a realizar as entrevistas em todas as regionais da cidade de Belo Horizonte (Barreiro, Centro-Sul, Leste, Nordeste, Noroeste, Norte, Oeste, Pampulha e Venda Nova). Os grupos de convivência de idosos dos CRAS(s) e o CRPI são programas de política social da Prefeitura de Belo Horizonte para atenção às pessoas idosas residentes na comunidade. A escolha destes locais de coleta dos dados foi devido ao perfil não institucionalizado dos participantes. O estudo foi autorizado pela coordenadora do CRPI e pelo Secretário Municipal Adjunto de Assistência Social da SMAAS.

O estudo foi divulgado no microfone ou, quando o grupo era menor, pessoalmente pela primeira autora ou pelos profissionais que trabalham no local. Os questionários foram aplicados por entrevista individual pela $1^{\mathrm{a}}$ autora, que recebeu formação da equipe que formou os entrevistadores que aplicaram o questionário na Europa. Os critérios de inclusão foram: idade $\geq 60$ anos, residir na cidade de Belo Horizonte e ser capaz de dar o consentimento livre e esclarecido. Com a indicação dos profissionais que trabalham no CRPI e nos grupos de convivência, foram excluídos os indivíduos não orientados no tempo e no espaço.

O instrumento utilizado para coleta dos dados foi elaborado em um dos grupos de trabalho do projeto europeu, financiado pelo $7^{\circ}$ Programa Quadro da Comissão Europeia, denominado "Reflecting the Positive diveRsities of European prIorities for reSearch and Measurement in end of life cAre (PRISMA)"31. O questionário analisa as preferências e prioridades para os cuidados de fim de vida, numa situação hipotética de doença grave, como o câncer, com menos de um ano de vida. É composto por duas partes, a primeira inclui 10 perguntas sobre as preferências e as prioridades em fim de vida e a segunda parte inclui questões sobre as caraterísticas sociodemográficas e as experiências com a doença, morte e morrer $^{32}$.

Neste estudo utilizou-se a versão adaptada para o Brasil ${ }^{32}$. Por recomendações de profissionais brasileiros com vasta experiência no cuidado com idosos e nos cuidados paliativos, houveram mudanças na versão brasileira do questionário em relação ao original ${ }^{32}$. No questionário brasileiro optamos por diversificar os exemplos de doença grave (não só o câncer), alargando o grupo de doenças crônicas graves e ameaçadoras à con- 
tinuidade da vida, tais como, a cardiopatia, a doença pulmonar obstrutiva crônica, a insuficiência renal e a doença de Alzheimer. Além disso, as entrevistas realizadas no Brasil foram realizadas com a técnica de administração por entrevista presencial. $\mathrm{Na}$ Europa $^{3}$, o questionário foi aplicado pelo telefone e, no Quênia ${ }^{33}$ foi administrado como inquérito de rua.

Nesse estudo serão analisadas as seguintes questões: Se você tivesse uma doença grave, e tivesse eventualmente menos de um ano de vida, (i) gostaria de ser informadola que o seu tempo de vida era limitado? (ii) gostaria de ser informado/a sobre os sintomas e problemas que provavelmente iria ter? (iii) gostaria de ser informado/a sobre as opções disponiveis para cuidados (serviços disponiveis, lugares onde poderia receber assistência, tratamentos e medicação) e como esses poderiam afetá-lo/la?. As quatro opções de respostas para as três perguntas foram: sim, sempre; sim, mas apenas se eu perguntasse; não; não sei.

Os dados coletados foram tratados no Microsoft Excel $2010^{\circledR}$ e SPSS Statistics $22.0^{\circledR}$. A normalidade das distribuições foi testada usando o teste Kolmogorov-Smirnov (estatística de teste: KS). No caso de distribuições não-normais, utilizaram-se os testes não paramétricos de comparação de medianas de duas amostras independentes (Mann-Whitney, estatística de teste: MW) e de comparação de medianas de mais do que duas amostras independentes (Kruskal-Wallis, estatística de teste: KW). No caso do teste Kruskal -Wallis apresentar diferenças significativas entre os grupos, são efetuadas comparações múltiplas entre os pares de grupo, de forma a detectar entre que grupos é que existem essas diferenças. No caso das variáveis contínuas não-normais, os dados são descritos pela mediana (Med) e pelo primeiro e terceiro quartis $\left(\left[\mathrm{Q}_{1}-\mathrm{Q}_{3}\right]\right)$.

Para descrever variáveis categóricas, utilizaram-se as frequências $\mathrm{N}(\%)$. De modo a determinar os fatores associados à preferência de ser sempre informado sobre o tempo de vida limitado, foi efetuada uma regressão logística. A associação bi-variada (ser informado sempre versus as outras opções de resposta) e cada uma das variáveis independentes (gênero, grupo etário, escolaridade, estado civil, religião, etnia, atividade profissional nos últimos 7 dias, rendimento atual, com quantos adultos vive, com quantas crianças vive, saúde geral, preferência pelo local de morte, local menos preferido de morte, experiência com doença e morte) foram analisadas. Para despistar possíveis variáveis de confundimento, foi realizada uma regressão logística multivariada, na qual foram incluídas como co-variáveis aquelas que demonstraram uma associação estatisticamente significativa com a variável dependente (ser informado sempre versus as outras opções de resposta) na regressão logística simples. Valores de $\mathrm{p}$ $\leq 0.05$ são considerados significativos.

O estudo foi aprovado pelo Comitê de Ética do Departamento de Ciências Sociais e Saúde da Faculdade de Medicina da Universidade do Porto/Portugal e pelo Comitê de Ética em Pesquisa da Secretaria Municipal de Saúde de Belo Horizonte (SMSA-BH). Para os participantes que aceitaram participar do estudo foi explicado o objetivo da pesquisa e todos tiveram tempo de fazer perguntas e esclarecer dúvidas. Após concordarem, todos os sujeitos da pesquisa assinaram o Termo de Consentimento Livre e Esclarecido.

\section{Resultados}

A maioria dos participantes são aposentados $(80.5 \%)$, declaram que o rendimento atual "dá para viver" (51.7\%) e são da religião católica (66.8\%). Do total de participantes, $53.5 \%$ avaliam a saúde geral como boa. Além disso, 19.0\% já receberam um diagnóstico de doença grave nos últimos cinco anos; e 66.0\% já estiveram envolvidos nos cuidados ou no apoio a um familiar/ amigo nos seus últimos meses de vida (Tabela 1).

\section{Preferências por informações: tempo de vida limitado, sintomas e problemas e opções de cuidados de saúde}

Os dados (Tabela 2) mostram que numa situação de doença grave, com menos de um ano de vida, a maioria das pessoas idosas $(74.0 \%)$ gostaria de ser sempre informada sobre o tempo de vida limitado. Quando juntamos as opções ["sim, sempre" e "sim, mas apenas se eu perguntasse"], observamos que $78.5 \%$ dos participantes gostariam de receber a informação sobre o prognóstico de vida. A opção de não ser informado foi a segunda mais escolhida (20.5\%).

A preferência por ser informado sobre o tempo de vida limitado (74.0\%) foi menor em relação à ser informado sobre os sintomas e problemas (89.3\%) e ser informado sobre as opções disponíveis para cuidados (serviços disponíveis, lugares onde poderia receber assistência, tratamentos e medicação) (96.3\%) (Tabela 2). A preferência por ser informado sobre os sintomas e problemas foi maior para os homens $(93.0 \%)$ do que para as mulheres (86.7\%). Em relação à ser 
Tabela 1. Caraterísticas dos participantes.

\begin{tabular}{|c|c|c|}
\hline Variáveis & $\mathbf{n}$ & $\%$ \\
\hline \multicolumn{3}{|l|}{ Grupo etário } \\
\hline 60-69 anos & 217 & 54.3 \\
\hline $70-79$ anos & 121 & 30.3 \\
\hline$\geq 80$ & 62 & 15.5 \\
\hline \multicolumn{3}{|l|}{ Gênero } \\
\hline Mulher & 241 & 60.3 \\
\hline Homem & 159 & 39.7 \\
\hline \multicolumn{3}{|l|}{ Escolaridade } \\
\hline Sem escolaridade formal & 30 & 7.5 \\
\hline Até 4 anos de estudo & 149 & 37.3 \\
\hline Até 8 anos de estudo & 54 & 13.5 \\
\hline Até 11 anos de estudo & 122 & 30.5 \\
\hline Ensino superior & 45 & 11.3 \\
\hline \multicolumn{3}{|l|}{ Estado civil } \\
\hline Solteiro & 61 & 15.3 \\
\hline Casado/união de fato & 167 & 41.8 \\
\hline Separado/divorciado & 50 & 12.5 \\
\hline Viúvo & 122 & 30.5 \\
\hline \multicolumn{3}{|l|}{ Religião } \\
\hline Católica Apostólica Romana & 267 & 66.8 \\
\hline Evangélica & 63 & 15.8 \\
\hline Espírita & 37 & 9.3 \\
\hline Outra & 11 & 2.8 \\
\hline Não tem & 22 & 5.5 \\
\hline \multicolumn{3}{|l|}{ Etnia } \\
\hline Branca & 114 & 28.5 \\
\hline Preta & 63 & 15.8 \\
\hline $\begin{array}{l}\text { Parda e outra (1: amarela; } 1 \text { : } \\
\text { indígena) }\end{array}$ & 223 & 55.8 \\
\hline \multicolumn{3}{|c|}{ Atividade profissional nos últimos 7 dias } \\
\hline Estudante & 26 & 6.5 \\
\hline $\begin{array}{l}\text { Desempregado (à procura de } \\
\text { emprego ou à espera de emprego, } \\
\text { mas não à procura de emprego) }\end{array}$ & 41 & 10.3 \\
\hline $\begin{array}{l}\text { Situação de doença ou } \\
\text { incapacidade }\end{array}$ & 5 & 1.3 \\
\hline Trabalho remunerado & 136 & 34.0 \\
\hline Aposentado & 323 & 80.8 \\
\hline Pensionista & 102 & 25.5 \\
\hline Doméstica & 34 & 8.5 \\
\hline Outra & 38 & 9.5 \\
\hline \multicolumn{3}{|l|}{ Rendimento atual } \\
\hline Muito difícil viver & 24 & 6.0 \\
\hline Difícil viver & 55 & 13.8 \\
\hline Dá para viver & 207 & 51.7 \\
\hline Permite viver confortavelmente & 114 & 28.5 \\
\hline \multicolumn{3}{|c|}{ Com quem vive: adultos (incluindo ele próprio) } \\
\hline Com 0 adulto (o próprio) & 76 & 19.0 \\
\hline Com 1 adulto & 153 & 38.3 \\
\hline Com 2 adultos & 98 & 24.5 \\
\hline Com 3 adultos & 39 & 9.8 \\
\hline Com 4 ou mais adultos & 34 & 8.5 \\
\hline
\end{tabular}

Tabela 1. Caraterísticas dos participantes.

\begin{tabular}{|c|c|c|}
\hline Variáveis & $\mathbf{n}$ & $\%$ \\
\hline \multicolumn{3}{|l|}{ Com quem vive: crianças } \\
\hline Nenhuma & 351 & 87.8 \\
\hline 1 criança & 34 & 8.5 \\
\hline 2 ou mais crianças & 15 & 3.8 \\
\hline \multicolumn{3}{|c|}{ Como avalia a sua saúde geral } \\
\hline Razoável & 76 & 19.0 \\
\hline
\end{tabular}

Muito boa

Preferência pelo local de morte

Própria (inclui: casa de um

familiar ou amigo $)^{*}$

$\begin{array}{lll}\text { Unidade de cuidados paliativos } & 71 & 17.8\end{array}$

Hospital- mas não numa unidade $\quad 86 \quad 21.5$

de cuidados paliativos

Instituição de longa permanência $\quad 34 \quad 8.5$

para idosos (ILPI)

Local menos preferido de

morte

$\begin{array}{lll}\text { Própria casa } & 60 & 15.0\end{array}$

$\begin{array}{lll}\text { Casa de um familiar ou amigo } \quad 88 \quad 22.0 & 88.0\end{array}$

$\begin{array}{lll}\text { Unidade de cuidados paliativos } & 8 & 2.0\end{array}$

Hospital- mas não numa unidade $\quad 85 \quad 21.3$

de cuidados paliativos

$\begin{array}{lll}\text { ILPI } & 159 \quad 39.8\end{array}$

Experiência com doença e morte

Familiar ou amigo diagnosticado $\quad 299 \quad 74.8$

com doença grave nos últimos

5 anos

Viveu de perto a morte de

familiar ou amigo nos últimos

5 anos

Recebeu diagnóstico de doença $\quad 76 \quad 19.0$

grave nos últimos 5 anos

Envolvido no apoio a familiar ou $\quad 264 \quad 66.0$

amigo nos últimos meses de vida

${ }^{*}$ ) Apenas 2 indivíduos escolheram a opção "casa de familiar ou amigo" como local de morte preferido.

informado sobre as opções disponíveis de cuidados, observou-se que a preferência dos homens (96.8\%) por informações continua superior à das mulheres (95.8\%).

A preferência por ser sempre informado sobre o tempo de vida limitado pode ser observada pela maioria dos homens $(83.0 \%)$ e das mulheres (68.0\%) de todos os grupos etários, sendo que a percentagem de homens que gostariam de ser informados é superior, em todos os grupos etários, em relação às mulheres (Tabela 2). Os resultados da regressão logística indicam que ser mulher é um fator protetor significativo relativo a querer 
Tabela 2. Preferências por informações: tempo de vida, sintomas e problemas e opções de cuidados; gênero e grupo etário.

\begin{tabular}{|c|c|c|c|c|c|c|c|}
\hline \multirow{2}{*}{$\begin{array}{r}\text { Gostaria de ser } \\
\text { informado sobre }\end{array}$} & \multicolumn{3}{|c|}{ Grupos etários, Homens } & \multicolumn{3}{|c|}{ Grupos etários, Mulheres } & \multirow{2}{*}{ Total } \\
\hline & $60-69$ & $70-79$ & $\geq 80$ & $60-69$ & $70-79$ & $\geq 80$ & \\
\hline \multicolumn{8}{|c|}{ Tempo de vida limitado } \\
\hline Sim, sempre & $79(84.9 \%)$ & $38(80.9 \%)$ & $15(78.9 \%)$ & $84(67.7 \%)$ & $55(74.3 \%)$ & $25(58.1 \%)$ & $296(74.0 \%)$ \\
\hline $\begin{array}{l}\text { Sim, mas } \\
\text { apenas se eu } \\
\text { perguntasse }\end{array}$ & $2(2.2 \%)$ & $0(-)$ & $1(5.3 \%)$ & $10(8.1 \%)$ & $2(2.7 \%)$ & $3(7 \%)$ & $18(4.5 \%)$ \\
\hline Não & $12(12.9 \%)$ & $8(17.0 \%)$ & $3(15.8 \%)$ & $27(21.8 \%)$ & $17(23.0 \%)$ & $15(34.9 \%)$ & $82(20.5 \%)$ \\
\hline Não sei & $0(-)$ & $1(2.1 \%)$ & $0(-)$ & $3(2.4 \%)$ & $0(-)$ & $0(-)$ & $4(1.0 \%)$ \\
\hline \multicolumn{8}{|c|}{ Sintomas e problemas } \\
\hline Sim, sempre & $86(92.5 \%)$ & $46(97.9 \%)$ & $16(84.2 \%)$ & $107(86.3 \%)$ & $70(94.6 \%)$ & $32(74.4 \%)$ & $357(89.3 \%)$ \\
\hline $\begin{array}{l}\text { Sim, mas } \\
\text { apenas se eu } \\
\text { perguntasse }\end{array}$ & $1(1.1 \%)$ & $1(2.1 \%)$ & $0(-)$ & $7(5.6 \%)$ & $2(2.7 \%)$ & $2(4.7 \%)$ & $13(3.3 \%)$ \\
\hline Não & $5(5.4 \%)$ & $0(-)$ & $3(15.8 \%)$ & $10(8.1 \%)$ & $2(2.7 \%)$ & $9(20.9 \%)$ & $29(7.3 \%)$ \\
\hline Não sei & $1(1.1 \%)$ & $0(-)$ & $0(-)$ & $0(-)$ & $0(-)$ & $0(-)$ & $1(0.3 \%)$ \\
\hline \multicolumn{8}{|c|}{ Opções disponíveis de cuidados } \\
\hline Sim, sempre & $90(96.8 \%)$ & $47(100 \%)$ & $17(89.5 \%)$ & $117(94.4 \%)$ & $73(98.6 \%)$ & $41(95.3 \%)$ & $385(96.3 \%)$ \\
\hline $\begin{array}{l}\text { Sim, mas } \\
\text { apenas se eu } \\
\text { perguntasse }\end{array}$ & $0(-)$ & $0(-)$ & $1(5.3 \%)$ & $3(2.4 \%)$ & $1(1.4 \%)$ & $2(4.7 \%)$ & $7(1.8 \%)$ \\
\hline Não & $3(3.2 \%)$ & $0(-)$ & $1(5.3 \%)$ & $4(3.2 \%)$ & $0(-)$ & $0(-)$ & $8(2.0 \%)$ \\
\hline Não sei & $0(-)$ & $0(-)$ & $0(-)$ & $0(-)$ & $0(-)$ & $0(-)$ & $0(-)$ \\
\hline
\end{tabular}

ser sempre informado sobre o tempo de vida limitado (OR=0.436: 95\% IC: 0.266-0.714) (Tabela 3). Além disso, escolher a casa de um familiar ou amigo como local menos preferido de morte é considerado um fator de risco significativo ao fato de se querer ser sempre informado sobre o tempo de vida limitado $(\mathrm{OR}=2.605$ : $95 \% \mathrm{IC}$ : 1.225-5.540; Ref: própria casa).

No modelo de regressão logística multivariada foram incluídas como variáveis independentes o gênero e o local menos preferido de morte, que continuaram a ser significativas (Tabela 4). Assim, os fatores associados significativamente com o fato de querer ser sempre informado sobre o tempo de vida limitado foram: ser mulher (OR= 0.446: 95\% CI:0.269-0.738; Ref: homem) e ter escolhido a casa de um familiar ou amigo como o local menos preferido de morte $(\mathrm{OR}=$ 2.423: 95\% CI:1.130-5.198; Ref: própria casa).

\section{Discussão}

Do nosso conhecimento, este é o primeiro estudo a investigar as preferências de pessoas idosas brasileiras, residentes na comunidade da cidade de
Belo Horizonte, por informações numa situação de doença grave, com menos de um ano de vida. Os dados mostram que a maioria dos participantes gostaria de ser sempre informada sobre o tempo de vida limitado (74.0\%). No entanto, esta preferência foi menor do que querer saber sobre os sintomas e os problemas decorrentes da doença $(89.3 \%)$ e as opções disponíveis para cuidados de saúde (96.3\%). Um estudo europeu de base populacional mostrou que a maioria dos participantes $(73.9 \%)$ quer ser sempre informada sobre o tempo de vida limitado ${ }^{3}$. No entanto, as análises por grupo etário mostram uma preferência maior dos idosos de Belo Horizonte, principalmente do grupo etário mais velho (60-69 anos: $75.1 \%$; $\geq 70$ anos: $72.7 \%$ ) do que dos europeus (60-69 anos: $74.2 \%$; $\geq 70$ anos: $67.5 \%)^{3}$.

Esses resultados indicam que os profissionais de saúde precisam estar equipados de conhecimentos e habilidades para estabelecer uma boa comunicação com os pacientes ${ }^{3,4}$. Além disso, precisam estar preparados para antecipar notícias sobre o prognóstico e para dar informações sobre a doença e os tratamentos disponíveis para os pacientes em terminalidade de vida ${ }^{3}$. É também fundamental saber identificar e respeitar 
Tabela 3. Regressão logística univariada. Variável dependente: ser informado sempre sobre o tempo de vida limitado.

\begin{tabular}{|c|c|c|}
\hline Fatores & OR [95\% IC] & p-value \\
\hline \multicolumn{3}{|l|}{ Grupo etário (ref: 60-69 anos) } \\
\hline 70-79 anos & $1.100[0.652-1.856]$ & 0.720 \\
\hline$\geq 80$ anos & $0.602[0.329-1.102]$ & 0.100 \\
\hline \multicolumn{3}{|l|}{ Gênero (ref: homem) } \\
\hline Feminino & $0.436[0.266-0.714]$ & $0.001^{*}$ \\
\hline \multicolumn{3}{|l|}{ Estado Civil: (ref: solteiro/a) } \\
\hline Casado(a) ou em União Estável & $1.357[0.696-2.648]$ & 0.370 \\
\hline Separado(a) ou Divorciado(a) & $0.902[0.395-2.055]$ & 0.805 \\
\hline Viúvo(a) & $0.960[0.485-1.902]$ & 0.908 \\
\hline \multicolumn{3}{|l|}{ Escolaridade (ref: s/escolaridade) } \\
\hline Até 4 anos de estudo & $0.584[0.209-1.634]$ & 0.306 \\
\hline Até 8 anos de estudo & $0.475[0.154-1.461]$ & 0.194 \\
\hline Até 11 anos de estudo & $0.518[0.183-1.462]$ & 0.214 \\
\hline Ensino Superior & $0.618[0.191-2.005]$ & 0.423 \\
\hline \multicolumn{3}{|l|}{ Religião (ref: Católica Apostólica Romana) } \\
\hline Evangélica & $1.393[0.727-2.670]$ & 0.318 \\
\hline Espírita Kardecista & $1.194[0.514-2.774]$ & 0.681 \\
\hline Outras & $5.969[0.775-45.976]$ & 0.086 \\
\hline Não tem religião & $1.353[0.482-3.797]$ & 0.566 \\
\hline \multicolumn{3}{|l|}{ Etnia (ref: branca) } \\
\hline Preta & $0.790[0.399-1.565]$ & 0.499 \\
\hline Parda & $1.030[0.612-1.732]$ & 0.912 \\
\hline Outras & $0.341[0.021-5.631]$ & 0.452 \\
\hline \multicolumn{3}{|l|}{ Rendimento atual (ref: muito difícil) } \\
\hline Difícil & $0.976[0.323-2.948]$ & 0.966 \\
\hline Dá para viver & $0.836[0.316-2.210]$ & 0.718 \\
\hline Permite viver confortavelmente & $1.187[0.426-3.307]$ & 0.743 \\
\hline \multicolumn{3}{|l|}{ Saúde geral (ref: muito ruim) } \\
\hline Razoável & $0.976[0.323-2.948]$ & 0.966 \\
\hline Boa & $0.836[0.316-2.210]$ & 0.718 \\
\hline Muito boa & $1.187[0.426-3.307]$ & 0.743 \\
\hline \multicolumn{3}{|c|}{ Familiar/amigo diag. com doença grave nos últimos 5 anos (ref: sim) } \\
\hline Não & $0.778[0.472-1.285]$ & 0.327 \\
\hline \multicolumn{3}{|c|}{ Viveu de perto a morte de familiar/amigo nos últimos 5 anos (ref: sim) } \\
\hline Não & $0.988[0.614-1.592]$ & 0.961 \\
\hline \multicolumn{3}{|c|}{ Recebeu diag. de doença grave nos últimos 5 anos (ref: sim) } \\
\hline Não & $0.859[0.479-1.539]$ & 0.609 \\
\hline \multicolumn{3}{|c|}{ Envolvido no apoio a familiar/ amigo nos últimos meses de vida (ref: sim) } \\
\hline Não & $1.376[0.846-2.238]$ & 0.198 \\
\hline \multicolumn{3}{|c|}{$\begin{array}{l}\text { Preferência pelo local de morte (ref: própria casa ou casa de familiar } \\
\text { ou amigo) }\end{array}$} \\
\hline Unidade de cuidados paliativos & $0.883[0.479-1.631]$ & 0.692 \\
\hline Hospital & $0.745[0.426-1.302]$ & 0.745 \\
\hline ILPI & $1.049[0.447-2.462]$ & 0.912 \\
\hline \multicolumn{3}{|c|}{ Local menos preferido de morte (ref: própria casa) } \\
\hline Em casa de um familiar ou amigo & $2.605[1.225-5.540]$ & $0.013^{*}$ \\
\hline Unidade de cuidados paliativos & $0.965[0.210-4.432]$ & 0.963 \\
\hline Hospital & $1.658[0.811-3.389]$ & 0.166 \\
\hline ILPI & $1.666[0.884-3.141]$ & 0.114 \\
\hline
\end{tabular}

*: significativo a um nível de significância de 5\%. 
Tabela 4. Regressão logística multivariada. Variável dependente: ser informado sempre sobre o tempo de vida limitado.

\begin{tabular}{lcc}
\hline \multicolumn{1}{c}{ Variáveis: } & OR [95\% IC] & p-value \\
\hline Gênero (ref: homem) & & \\
$\quad$ Mulher & $0.446[0.269-0.738]$ & $\mathbf{0 . 0 0 2}^{*}$ \\
Local menos preferido de morte (ref: própria casa) & & \\
Casa de um familiar ou amigo & $2.423[1.130-5.198]$ & $\mathbf{0 . 0 2 3}^{*}$ \\
Unidade de cuidados paliativos & $1.059[0.228-4.925]$ & 0.942 \\
Hospital - mas não numa unidade de cuidados paliativos & $1.497[0.724-3.095]$ & 0.276 \\
ILPI & $1.415[0.739-2.706]$ & 0.295 \\
\hline
\end{tabular}

*: significativo a um nível de significância de 5\%.

as preferências daqueles que não querem ser informados, que no estudo de Belo Horizonte foi a segunda opção mais escolhida pelos participantes $(20.5 \%)$. Deve-se prover suporte para os profissionais de saúde por meio de treinamentos sobre comunicação em fim de vida e incluir nos currículos acadêmicos disciplinas que abordem o tema dos cuidados paliativos ${ }^{4}$. É ainda relevante promover campanhas para esclarecer a população brasileira sobre questões que envolvem a morte e morrer e sobre cuidados paliativos e incentivá-las a conversar com os clínicos sobre as suas vontades e preferências em fim de vida.

Estudos sugerem que a preferência por querer receber a informação depende do tipo de população estudada ${ }^{14,34}$. Alguns países tradicionalmente católicos, tal como a Itália, têm sido associados a maior resistência em comunicar a informação completa para o paciente gravemente doente e com uma medicina de tradição paternalista ${ }^{3,35}$. Outros estudos mostram que as preferências por informações prognósticas podem ser influenciadas por grupos étnicos e raciais ${ }^{34}$. No entanto, os nossos dados mostram que apesar da maioria dos participantes se declarar católicos $(66.8 \%)$ e ainda ser comum encontrar no Brasil médicos com uma postura paternalista em relação aos seus pacientes $^{7,36}$, a maioria das pessoas idosas quer sempre ser informada sobre o tempo de vida limitado. Uma das explicações para esse resultado pode ter sido devido ao perfil das pessoas idosas que participaram do estudo. O estudo foi realizado em locais onde são oferecidos às pessoas idosas diversos serviços, incluindo atividades físicas, alfabetização e informática, e programas para garantir a promoção e defesa dos seus direitos. Assim, as pessoas idosas que frequentam esses locais têm um perfil mais ativo e que cuidam da sua saúde e têm saúde para o fazer. Talvez as pessoas idosas com outro perfil, tal como mais dependentes fisicamente, podem ter outras preferências. No entanto, essas relações não estão claras. Embora a evidência mostre que a divulgação do prognóstico é fundamental nos cuidados com pessoas idosas, faltam estudos sobre as preferências por informações nessa população ${ }^{34}$.

$O$ gênero pode exercer um papel significativo nas preferências pela informação e tomada de decisões em saúde ${ }^{37}$. Os homens foram mais propensos a querer receber informações sobre o tempo de vida limitado do que as mulheres. Estudos anteriores também encontraram uma preferência maior dos homens por informações prognósticas do que das mulheres ${ }^{3,38}$. Alguns estudos mostram que mulheres com câncer avançado são mais prováveis de ter um papel mais ativo nas decisões em saúde mas desejam maior apoio emocional dos seus oncologistas ${ }^{38,39}$. Por outro lado, os pacientes homens têm um papel mais passivo na tomada de decisão mas desejam receber mais informações sobre sua saúde ${ }^{38,39}$. Além disso, sabe-se que o gênero é um fator que influencia a maneira como as pessoas idosas envelhecem e a sua relação com os cuidados com à saúde ${ }^{40}$. Sendo assim, homens e mulheres podem ter percepções e comportamentos diferentes relacionadas aos cuidados de saúde no final da vida.

Os resultados também indicam que aqueles que escolheram a opção "casa de um familiar ou amigo" como local de morte menos preferido foram mais propensos a escolher ser sempre informados sobre o tempo de vida limitado do que aqueles que escolheram a "própria casa". Os nossos dados mostram que a maioria das pessoas idosas quer participar da tomada de decisão em saúde tanto num cenário de capacidade de tomar decisões $(95.3 \%)$ como de incapacidade $(64.5 \%)^{41}$. Talvez aqueles que escolheram a opção "casa de um familiar ou amigo" têm mais receio de que neste local exista uma menor possibilida- 
de de exercer sua autonomia para tomar decisões e, assim, desejam mais informações do que aqueles que escolheram a "própria casa", que é um local mais provável de ter suas preferências respeitadas. No entanto, as evidências sobre o local de morte, especialmente com a população idosa, ainda não está claro e menos evidência existe sobre o local menos preferido de morte ${ }^{2,42,30}$. Sendo assim, são necessários mais estudos para analisar a influência desses fatores nas preferências.

Este estudo apresenta algumas limitações. Como referido anteriormente, as pessoas idosas com mais dependência funcional e fragilidade podem ter sido sub-representadas. Sendo assim, estudos futuros devem incluir participantes com maior dependência funcional. Nenhum dos participantes avaliou a sua saúde geral como ruim ou muito ruim, sendo assim, é possível que alguns tenham dificuldade em imaginar uma situação de doença grave, com menos de um ano de vida. No entanto, $19.0 \%$ dos participantes de Belo Horizonte já tinham enfrentado uma doença grave nos últimos cinco anos e $\geq 66.0 \%$ já tiveram, nos últimos cinco anos, algum familiar próximo/amigo com diagnóstico de doença grave, ou viveram de perto a morte de familiar/ amigo ou estiveram envolvidos nos cuidados ou apoio a algum familiar/amigo nos últimos meses de vida. Sendo assim, as experiências dos participantes com a doença grave e a morte ajudaram a contextualizar o cenário hipotético utilizado no questionário. Recomenda-se que, no futuro, sejam realizados estudos com pessoas idosas com doença avançada para verificar se as preferências por informações mudam quando são confrontadas com um diagnóstico ruim.

\section{Conclusão}

Os resultados do estudo mostram que a maioria das pessoas idosas de Belo Horizonte, residentes na comunidade, quer ser informada sobre o tempo de vida limitado numa situação de doença avançada. Os homens são mais propensos de querer receber informações prognósticas do que as mulheres. Os resultados deste estudo são um alerta para os profissionais de saúde que cuidam de pessoas idosas com doença grave e com prognóstico limitado. É fundamental estabelecer uma comunicação eficaz com o paciente, o mais precocemente possível, para que as suas preferências sejam comunicadas e respeitadas.

\section{Colaboradores}

R Jorge participou da concepção, delineamento, coleta, análise e interpretação dos dados e da redação do artigo. A Teixeira participou da análise e interpretação dos dados e da revisão do artigo. N Calanzani participou da análise e interpretação dos dados e da revisão do artigo. $R$ Nunes participou da concepção e do delineamento e da revisão crítica da versão a ser publicada. L Sousa participou da concepção e do delineamento, da análise e interpretação dos dados e da revisão crítica da versão a ser publicada. 


\section{Agradecimentos}

Aos pesquisadores do Projeto PRISMA, em especial à Bárbara Gomes e Natália Calanzani (Cicely Saunders Institute, King's College London) pela autorização para a adaptação do questionário no Brasil e pelo apoio material e incentivo à pesquisa. O Projeto PRISMA foi financiado pelo Sétimo Programa-Quadro da Comissão Europeia com o objetivo de coordenar investigação internacional de alta qualidade em cuidados de fim de vida para doentes oncológicos. Investigador Principal: Richard Harding. Diretora Científica: Irene J Higginson. Outros membros do PRISMA: Gwenda Albers, Barbara Antunes, Ana Barros Pinto, Claudia Bausewein, Dorothea Bechinger-English, Hamid Benalia, Emma Bennett, Lucy Bradley, Lucas Ceulemans, Barbara A Daveson, Luc Deliens, Noël Derycke, Martine de Vlieger, Let Dillen, Julia Downing, Michael Echteld, Natalie Evans, Dagny Faksvåg Haugen, Silvia Finetti, Nancy Gikaara, Barbara Gomes, Marjolein Gysels, Sue Hall, Richard Harding, Irene J Higginson, Stein Kaasa, Jonathan Koffman, Pedro Lopes Ferreira, Arantza Meñaca, Johan Menten, Natalia Monteiro Calanzani, Fliss Murtagh, Bregje OnwuteakaPhilipsen, Roeline Pasman, Francesca Pettenati, Robert Pool, Richard A. Powell, Miel Ribbe, Katrin Sigurdardottir, Steffen Simon, Franco Toscani, Bart Van den Eynden, Paul Vanden Berghe e Trudie van Ierse.

$\mathrm{R}$ Jorge foi apoiada por 14 meses por uma bolsa de doutorado no exterior pela Coordenação de Aperfeiçoamento de Pessoal de Nível Superior (CAPES). A Teixeira agradece o financiamento através da bolsa de investigação referente ao projeto "NanoSTIMA: Macro-to-Nano Human Sensing: Towards Integrated Multimodal Health Monitoring and Analytics/NORTE-010145-FEDER-000016", financiado pelo programa NORTE 2020, através do PORTUGAL 2020 e dos Fundos Europeus para o Desenvolvimento Regional (ERDF).

L Sousa foi apoioada por Fundos Nacionais através da FCT - Fundação para a Ciência e a Tecbologia no CINTESIS, R\&D Unit.

\section{Referências}

1. Hall S, Petkova H, Tsouros AD, Costantini M, Higginson IJ. Palliative Care for Older People: Better Practices. Copenhagen: WHO Regional Office for Europe; 2011.

2. Gomes B, Calanzani N, Gysels M, Hall S, Higginson IJ. Heterogeneity and changes in preferences for dying at home: a systematic review. BMC Palliat Care 2013;12(1):7.

3. Harding R, Simms V, Calanzani N, Higginson IJ, Hall S, Gysels M, Meñaca A, Bausewein C, Deliens L, Ferreira P, Toscani F, Daveson BA, Ceulemans L, Gomes $B$, PRISMA. If you had less than a year to live, would you want to know? A seven-country European population survey of public preferences for disclosure of poor prognosis. Psychooncology 2013;22(10):22982305.

4. Sleeman K. End-of-life communication: let's talk about death. J R Coll Physicians Edinb 2013;43(3):197199.

5. Alelwani SM, Ahmed YA. Medical training for communication of bad news: A literature review. J Educ Health Promot 2014;3:51.

6. Barnett MM, Fisher JD, Cooke H, James PR, Dale J. Breaking bad news: consultants' experience, previous education and views on educational format and timing. Med Educ 2007;41(10):947-956.

7. Fumis RRL, De Camargo B, Del Giglio A. Physician, patient and family attitudes regarding information on prognosis: A Brazilian survey. Ann Oncol 2012;23(1):205-211.

8. Mitchell AJ. Reluctance to disclose difficult diagnoses: A narrative review comparing communication by psychiatrists and oncologists. Support Care Cancer 2007;15(7):819-828.

9. Repetto L, Piselli P, Raffaele M, Locatelli C. Communicating cancer diagnosis and prognosis: When the target is the elderly patient-a GIOGer study. Eur J Cancer 2009;45(3):374-383.

10. Wenrich MD, Curtis JR, Shannon SE, Carline JD, Ambrozy DM, Ramsey PG. Communicating with dying patients within the spectrum of medical care from terminal diagnosis to death. Arch Intern Med 2001;161(6):868-874.

11. Shahidi J. Not telling the truth: circumstances leading to concealment of diagnosis and prognosis from cancer patients. Eur J Cancer Care (Engl) 2010;19(5):589593.

12. De Vleminck A, Pardon K, Roelands M, Houttekier D, Van den Block L, Vander Stichele R, Deliens L. Information preferences of the general population when faced with life-limiting illness. Eur J Public Health 2015;25(3):532-538.

13. Cartwright LA, Dumenci L, Siminoff LA, Matsuyama RK. Cancer patients' understanding of prognostic information. J Cancer Educ 2014;29(2):311-317.

14. Elkin EB, Kim SHM, Casper ES, Kissane DW, Schrag D. Desire for information and involvement in treatment decisions: Elderly cancer patients' preferences and their physicians' perceptions. J Clin Oncol 2007;25(33):5275-5280.

15. Mack JW, Cronin A, Keating NL, Taback N, Huskamp HA, Malin JL, Earle CC, Weeks JC. Associations between end-of-life discussion characteristics and care received near death: a prospective cohort study. J Clin Oncol 2012;30(35):4387-4395. 
16. Hagerty RG. Communicating prognosis in cancer care: a systematic review of the literature. Ann Oncol 2005;16(7):1005-1053.

17. Parker SM, Clayton JM, Hancock K, Walder S, Butow PN, Carrick S, Currow D, Ghersi D, Glare P, Hagerty R, Tattersall MH. A systematic review of prognostic/ end-of-Life communication with adults in the advanced stages of a life-limiting illness: patient/caregiver preferences for the content, style, and timing of information. J Pain Symptom Manage 2007;34(1):81-93.

18. Tang ST, Lee S-YC. Cancer diagnosis and prognosis in Taiwan: Patient preferences versus experiences. Psychooncology 2004;13(1):1-13.

19. Price EL, Bereknyei S, Kuby A, Levinson W, Braddock $\mathrm{CH}$. New elements for informed decision making: A qualitative study of older adults' views. Patient Educ Couns 2012;86(3):335-341.

20. World Health Organization (WHO), World Palliative Care Alliance. Global Atlas of Palliative Care at the End of Life. Geneva: WHO; 2014.

21. Paillaud E, Canoui-Poitrine F, Varnier G, Anfasi-Ebadi N, Guery E, Saint-Jean O, Gisselbrecht M, Aparicio T, Bastuji-Garin S, Laurent M, Caillet P. Preferences about information and decision-making among older patients with and without cancer. Age Ageing 2017;46(4):665-671.

22. Ajaj A, Singh MP, Abdulla AJ. Should elderly patients be told they have cancer? Questionnaire survey of older people. BMJ 2001;323(7322):1160.

23. Jenkins V, Fallowfield L, Saul J. Information needs of patients with cancer: Results from a large study in UK cancer centres. Br J Cancer 2001;84(1):48-51.

24. Giacalone A, Blandino M, Talamini R, Bortolus R, Spazzapan S, Valentini M, Tirelli U. What elderly cancer patients want to know? Differences among elderly and young patients. Psychooncology 2007;16(4):365370.

25. Fried TR, Bradley EH, O'Leary J. Prognosis communication in serious illness: Perceptions of older patients, caregivers, and clinicians. J Am Geriatr Soc 2003;51(10):1398-1403.

26. Chouliara Z. Perceptions of older people with cancer of information, decision making and treatment: a systematic review of selected literature. Ann Oncol 2004;15(11):1596-1602.

27. Cassileth BR, Zupkis RV, Sutton-Smith K, March V. Information and participation preferences among cancer patients. Ann Intern Med 1980;92(6):832-836.

28. Levinson W, Kao A, Kuby A, Thisted RA. Not all patients want to participate in decision making:A national study of public preferences. J Gen Intern Med 2005;20(6):531-535.

29. Krejcie RV, Morgan DW. Determining Sample Size for Research Activities. Educ Psychol Meas 1970; 30(3):607-610.

30. Jorge R, Calanzani N, Freitas A, Nunes R, Sousa L. Preference for death at home and associated factors among older people in the city of Belo Horizonte, Brazil. Ciên Saude Colet 2019;24(8):3003-3014.

31. Ferreira PL, Antunes B, Pinto AB, Gomes B. Cuidados de fim de vida: Portugal no projeto europeu PRISMA. Rev Port Saude Publica 2012;30(1):62-70.
32. Jorge R, Sousa L, Nunes R. Preferências e prioridades para os cuidados de fim de vida de pessoas idosas: adaptação cultural para o Brasil. Geriatr Gerontol Aging 2016;10(2):101-111.

33. Downing J, Gikaara N, Gomes B, Daveson BA, Higginson IJ, Harding R. Public opinion on preferences and priorities for end-of-life care in sub-Saharan Africa: piloting a novel method of street surveying. BMJ Support Palliat Care 2012;2(1):72-74.

34. Ahalt C, Walter LC, Yourman L, Eng C, Pérez-Stable EJ, Smith AK. "Knowing is better": Preferences of diverse older adults for discussing prognosis. J Gen Intern Med 2012;27(5):568-575.

35. Toscani F. Deception, catholicism, and hope: Understanding problems in the communication of unfavorable prognoses in traditionally-catholic countries. Am J Bioeth 2006;6(1):W6-W18.

36. Moritz RD, Deicas A, Capalbo M, Forte DN, Kretzer LP, Lago P, Pusch R, Othero J, Piva J, Silva NB, Azeredo N, Ropelato R. II Fórum do "Grupo de Estudos do Fim da Vida do Cone Sul”: definições, recomendações e ações integradas para cuidados paliativos na unidade de terapia intensiva de adultos e pediátrica. Rev Bras Ter Intensiva 2011;23(1):24-29.

37. Fletcher K, Prigerson HG, Paulk E, Temel J, Finlay E, Marr L, McCorkle R, Rivera L, Munoz F, Maciejewski PK. Gender differences in the evolution of illness understanding among patients with advanced cancer. $J$ Support Oncol 2013;11(3):126-132.

38. Marwit SJ, Datson SL. Disclosure preferences about terminal illness: An examination of decision-related factors. Death Stud 2002;26(1):1-20

39. Gaston CM, Mitchell G. Information giving and decision-making in patients with advanced cancer: A systematic review. Soc Sci Med 2005;61(10):2252-2264

40. Silva SPC, Menandro MCS. As representações sociais da saúde e de seus cuidados para homens e mulheres idosos. Saude e Soc 2014;23(2):626-640.

41. Jorge R. Older people's preferences and priorities for end of life care in the city of Belo Horizonte, MG, Brazil [tese]. Aveiro: Universidade de Aveiro, Porto: Instituto de Ciências Biomédicas Abel Salazar, Universidade do Porto; 2017.

42. Calanzani N, Moens K, Cohen J, Higginson IJ, Harding R, Deliens L, Toscani F, Ferreira PL, Bausewein C, Daveson BA, Gysels M, Ceulemans L, Gomes B, Project PRISMA. Choosing care homes as the least preferred place to die: a cross-national survey of public preferences in seven European countries. BMC Palliat Care 2014;13(1):48.

Artigo apresentado em 28/01/2018

Aprovado em 16/04/2018

Versão final apresentada em 18/04/2018 
